\title{
An information-based solution for the puzzle of testimony and trust
}

\author{
Mariarosaria Taddeo \\ University of Hertfordshire \\ mariarosaria.taddeo@philosophyofinformation.net
}

\begin{abstract}
In this paper, I offer a contribution to the debate on testimony that rests on three elements: the definition of semantic information (Floridi, 2004), the analysis of trust as a second-order property of first-order relations provided in (Taddeo, 2010), and Floridi's Network Theory of Account (NTA) (Floridi, Forthcoming). I argue that testimony transmits semantic information and it is neither grounded on trust nor is it justified by it. Instead, I show that testimony is an occurrence of a first-order relation of communication affected by the second-order property of trust. I then defend the view that an epistemic agent can acquire some knowledge, on the basis of the information communicated through testimony, if and only if the agent is able to connect the transmitted information to the conceptual network of interrelation to which it belongs. I refer to Floridi's NTA to show how such a network allows the epistemic agent to achieve knowledge on the basis of semantic information.
\end{abstract}

Key words: Justification, Knowledge, Network Theory of Account, Semantic Information, Testimony, Trust. 


\section{An information-based solution for the puzzle of testimony and trust}

\section{Introduction}

Testimony is commonly understood as the assertion of a declarative sentence carrying the message of a sender (S) to a receiver (R), ${ }^{1}$ who then accepts it as true, without checking its truthfulness. ${ }^{2}$ From an epistemological point of view, true beliefs acquired through testimony are not (yet) justified, because they (still) lack any verification of their truthfulness. For this reason, testimony has been traditionally considered an inferior source of beliefs and of knowledge by, among others, Plato and Locke. This is problematic, because, on the basis of this approach, we would have to reject one of the most common ways to share epistemic content in social systems. Such a problem could be overcome by showing that testimony can be a source of knowledge. The problem of knowledge achievement (henceforth the KA problem) then arises, that is, whether it is possible to achieve any knowledge at all through testimony.

This paper contributes to the debate on testimony by suggesting a new perspective to address and solve the KA problem. In a testimony scenario, there are three fundamental aspects that deserve attention: the message, the presence of trust, and the knowledge that the receiver of the message might achieve. I will argue that the solution of the KA problem rests on a three-phase process, where each phase is devoted to clarifying the nature of one of these aspects.

In what follows, I will first analyse the nature of the message (M) conveyed through testimony by endorsing an information-based perspective. Then I will focus on the relation between trust and testimony. In a testimony scenario, $\mathrm{R}$ accepts S' message on the basis of R's trust in $\mathrm{S}$. As illustrated in Figure 1, $\mathrm{R}$ is the trustor, who accepts $\mathrm{S}$ ' message as true without verifying it and only on the basis of his trust in S, while $\mathrm{S}$ is the trustee: she is the referent of R's trust.

\section{INSERT FIGURE 1 HERE}

Figure 1 The link between trust and testimony scenarios.

\footnotetext{
${ }^{1}$ In this paper I will refer to 'sender and receiver' of a message rather than to 'speaker and hearer' to indicate the agents involved in a testimony scenario. 'Hearer and receiver', although common terms in the philosophical literature on testimony, specifically refer to verbal communication, which is only one of the possible way in which testimony can occur, while 'sender and receiver' generally refer to a case of communication among agents, without specifying the nature of the communication. For this reason the latter is more appropriate to describe possible testimony scenarios.

2 (Coady, 1992), (Graham, 1997), (Goldberg, 2001), (Lackeyand \& Sosa, 2006), (Pritchard, 2004).
} 
Many of the contributions to the debate on testimony agree in considering testimony linked in some way to trust. ${ }^{3}$ Trust has been considered the source of epistemic justification for the receiver of the message to believe the communicated message to be true. ${ }^{4}$ This is already a problematic thesis in itself. But even assuming that it might be accepted, other problems arise, since one has not explained yet whether and how trust can provide such a justification, and what the reasons are that justify R's decision to trust $\mathrm{S}$ is transmitting true messages.

The debate on testimony is wide and heterogeneous, and different analyses have been provided from a variety of points of view to answer these questions. ${ }^{5}$ I will analyse the relation between testimony and trust on the basis of the definition of trust as a second-order property of first-order relations proposed in (Taddeo, 2010). This analysis will show that, while the presence of trust is a necessary condition for the occurrence of testimony, trust should not be considered the source of epistemic justification for $\mathrm{R}$ to consider that $\mathrm{S}$ ' message is true. This will conclude the second phase of the KA solution process.

Once the first two phases are completed, I will introduce Floridi's Network Theory of Account (NTA) (Floridi, Forthcoming). Floridi provides an information-based analysis of knowledge, according to which knowledge is "accounted semantic information" (Floridi, Forthcoming). I will show that the NTA provides the necessary conceptual tools to complete the third phase and to solve the KA problem.

Now that the plan has been outlined, we may proceed to analyse in detail the nature of the message conveyed through testimony.

\section{An informational perspective for testimony}

$\mathrm{M}$ is commonly considered as a way of conveying S' belief, which, if true and justified, allows $\mathrm{R}$ to acquire knowledge of it.

If one considers the wide and heterogeneous range of occurrences of testimony, which varies depending on the context and on the way in which a message is communicated, then the definition of $\mathrm{M}$ as a doxastic propositional content becomes immediately too restrictive. Such a definition excludes all those cases in which testimony does not transmit $S$ ' beliefs but some other sort of content. Consider, for example, those cases in which $\mathrm{S}$ communicates a message that $\mathrm{S}$ does

\footnotetext{
${ }^{3}$ See for example (Blais, 1987), (Hardwig, 1991), (Welbourne, 1981, 1993), (Schmitt, 1987), (Goldman, 1999), (Baier, 1994), and (Jones, 1999).

4 (Baier, 1995), (Govier, 1993), (Hinchman, 2005), (Holton, 1994), (Lagenspetz, 1992), (McDowell, 2002), (Burge, 1997), (Govier, 1997).

${ }_{5}$ Consider for example the debate between reductionism and anti-reductionism, (Audi, 1997), (Graham, 2000) and (Fricker, 1995) or the contributions provided within the framework of social epistemology and of feminist epistemology (Antony \& Witt, 1993).
} 
not understand, and about which S does not hold any belief. Imagine the case of a spy, who learns by heart a chemistry formula which he does not understand. Imagine that the spy communicates the formula to someone, who perfectly understands it and who, thanks to the spy's testimony, acquires knowledge of a new chemical process. In this case, despite the fact that $\mathrm{S}$ does not hold any belief about $\mathrm{M}, \mathrm{R}$ understands the communicated message and is also able to achieve some knowledge on the basis of S' communication.

If testimony occurs also in those circumstances in which $\mathrm{S}$ does not hold a belief about $\mathrm{M}$, then we have to conclude that $\mathrm{M}$ does not necessarily transmit a doxastic propositional content. Such a conclusion leads to a new problem concerning M's nature.

The nature of the message becomes clear if one considers the requirements that $\mathrm{M}$ needs to satisfy in order for testimony to occur. They are at least two: M must be meaningful and truthful. M must be meaningful, because testimony is a form of communication, and it is successful only when $M$ has a meaning that is understood by the intended receiver (Littlejohn, 2002). M must also be truthful with respect to its reference because, if $\mathrm{M}$ is false predicatively, that is, not true, then testimony becomes false testimony attributively, that is, not a genuine form of testimony at all. In order to transmit some meaningful message, testimony has to convey some semantic content.

The second requirement specifies that the transmitted semantic content must be true. It turns out that testimony conveys semantic information (henceforth information) (Floridi, 2004), that is well-formed, meaningful and veridical data. According to this definition, information is intrinsically true, and is substantially different from well-formed, meaningful but false data, which qualify as dis-information or mis-information. ${ }^{6}$ While information is the content communicated in genuine cases of testimony, mis- and dis-information should be considered the content conveyed in nongenuine testimony cases. In the rest of this paper, I will focus only on the genuine occurrences of testimony.

The reader should notice that the analysis presented in this section endorses a minimalist approach, according to which the analysis of M's nature has been developed by considering only the necessary and sufficient conditions that $\mathrm{M}$ needs to satisfy in a genuine testimony scenario. This does not exclude the possibility of different scenarios, in which $\mathrm{M}$ might transmit something more complex than an instance of information and, for example, convey either $S$ ' true beliefs or some knowledge that $\mathrm{S}$ has. These scenarios do not contradict the analysis presented in this section, because in both cases M's content can be defined in informational terms - as an instance of

\footnotetext{
${ }^{6}$ The intrinsic veridical nature of information is at the centre of a wide debate, which is out of the scope of this paper. The reader interested in knowing more about the debate on the alethic nature of information is referred to (Bar-Hillel \& Carnap, 1953), (Devlin, 1991), (Colburn, 2000) for the arguments against the veridical nature of information and to
} 
information processed by a cognitive system (belief) or as a justifiable or explainable instance of information (knowledge). Hence, also in these cases, M's content remains consistent with the definition presented above. With this analysis of M's nature in mind, we may now turn to the second phase and focus on the relation between testimony and trust.

\section{The puzzle of testimony and trust}

Trust is often understood as a relation that holds when (a) one of the parts (the trustor) chooses to rely on another part (the trustee) to perform a given action, and (b) this choice rests on the assessment of the trustee's trustworthiness. The trustor's appraisal of the trustee's trustworthiness is usually considered the foundation of trust (Gambetta, 1998; Taddeo, 2009). Trustworthiness is defined as the set of beliefs that the trustor holds about the potential trustee's abilities and performances, and the probabilities he assigns to those beliefs (Gambetta, 1998).

When analysed in relation to testimony, trust has been considered the source of epistemic justification for $\mathrm{R}$ to believe $\mathrm{M}$ to be true without verifying it. ${ }^{7} \mathrm{~A}$ careful analysis of the testimony scenario shows that R's trust in S explains R's decision to accept as true the message communicated by $\mathrm{S}$, but such an explanation is insufficient to provide an epistemic justification. Consider those cases in which R's trust is grounded on faith, superstition, ignorance or random decision. In all these circumstances, the presence of trust allows for understanding R's decision but does not provide any justification for R's choice to accept S' message as true. Admittedly, in these circumstances, even if $\mathrm{S}$ communicated a true message to $\mathrm{R}$, we would not ascribe any knowledge of $\mathrm{M}$ to $\mathrm{R}$.

This shows that considering trust as the source of epistemic justification does not solve the KA problem, it rather leads to a more fundamental question about the justification of R's decision to trust. In the best possible scenario, R's trust is grounded on (and justified by) rational criteria and objective evidence of S' trustworthiness. Nevertheless, also in these circumstances trust does not provide an epistemic justification for R's choice, because even when trust is grounded on rational and objective criteria one cannot exclude the occurrence of epistemic luck. Suppose, for example, that Sam and Robert are students in the same class, and that they have known each other for a long time. Sam has always been the best student in the class and she has the reputation of remembering the dates of all the important events in history of science, like the discovery of Radium. Suppose that Sam asserts to Robert that Radium was discovered in 1898, but that this is

(Dretske, 1981), (Grice, 1989), (Floridi, 2004) and (Floridi, 2005) for the arguments in favour of the veridical nature of information.

7 (Baier, 1995), (Govier, 1993), (Hinchman, 2005), (Holton, 1994), (Lagenspetz, 1992), (McDowell, 2002), (Burge, 1997), (Govier, 1997). 
the one event where Sam does not know the year it occurs. She just asserts it to impress Robert and she is lucky to pick the right year. Robert trusts Sam on the basis of a rational and objective analysis, i.e. she always knows the year of an event and she is the best student in the class. Still the message communicated by Sam is true only by chance.

Trust is a decision that always involves some risks for the trustor that the trustee could behave differently from what she is expected to do, e.g. knowing the year of any given event in the history of science. The risk can be reduced when the trustee is chosen on the basis of rational and objective criteria, but in these cases too, trust entails some (albeit potentially low) risks. The presence of these risks does not allow for excluding epistemic luck a priori. For this reason, it is impossible to rule out the possibility that $\mathrm{R}$ might have been sent a truthful message just by chance when the justification for accepting the communicated message rests on R's trust. We have then to conclude that trust cannot be considered the source of epistemic justification and that the means for acquiring knowledge through testimony lay somewhere else than trust.

Despite it not being the source of epistemic justification, trust is certainly present in testimony scenarios. It remains to be clarified what the relation between testimony and trust is. The rest of this section focuses on this relation and explains it on the basis of the analysis of trust presented in (Taddeo, 2010).

According to this analysis, the occurrences of trust are related to, and affect, pre-existing relations, like purchasing, negotiation, communication and delegation. Trust is not to be considered a relation itself but a property of relations, something that changes the way relations occur. Consider, for example, a case of communication. In the late thirteenth century, the Venetian explorer Marco Polo came back from a journey that took him to the Far East. ${ }^{8}$ After his return, he was arrested and, while in jail, he narrated his adventures to a fellow prisoner, who wrote them down in a book entitled The Million. The book became famous in the West and was the means by which Europeans could learn about the East. In this case, there are two links between the reader of Marco Polo's book and Marco Polo. There is a first-order relation, the communication, which ranges over the two agents, and there is the second-order property of trust that ranges over the first-order-relation and affects the way it occurs.

As a property of relations, trust affects the way relations occur by minimising the trustor's effort and commitment for the achievement of a given goal. It does so in two ways. First, the trustor can avoid performing the action necessary to achieve his goal himself, because he can count on the trustee to do it (or have done it). This is true even in epistemic contexts in which the trustor, e.g. a member of a jury, could not physically replace the trustee, e.g. an eyewitness. Second, the trustor 
can decide not to supervise the trustee's performance. This is a peculiarity of trust scenarios (Taddeo, 2010). The reader should note that, in those cases in which the trustee's trustworthiness has been objectively assessed, the trustor takes a low risk with regard to the trustee's noncompliance. In these cases, the absence of supervision is grounded on the trustee's trustworthiness, which is a measure of the probability that the trustee is able to perform a given action correctly and autonomously. Nevertheless, the criteria for the assessment of trustworthiness vary from case to case, and may not be rational or objective. In these cases, the trustor still does not supervise the trustee, even though this entails some high risks.

The analysis presented so far clarifies the role of trust in the case of the readers of The Million, who did not have to travel to China to know about that country. The minimisation of the effort and commitment determined by the presence of trust allowed them to save resources (especially time and energy) that they would have spent in performing the action that Marco Polo, the trustee, executed, and in supervising his performance. ${ }^{9}$ It follows that trust can be defined thus:

Trust: Assume a set of first order-relations functional to the achievement of a goal and that two agents are involved in the relations, such that one of them (the trustor) has to achieve the given goal while the other (the trustee) is able to perform some task in order to achieve that goal. If the trustor chooses to achieve his goal through the task performed by the trustee, and if the trustor considers the trustee a trustworthy agent, then the relation has the property of being advantageous for the trustor. Such a property is a second-order property that affects the first-order relations occurring between agents and is called trust. ${ }^{10}$

This definition of trust casts a new light on the occurrences of testimony and allows for reconsidering the relation between testimony and trust. As shown in section two, in a testimony scenario, S transmits some information to R, so testimony is an instance of communication, a firstorder relation. This is a partial definition, because it does not take into consideration other aspects of testimony, such as the goal of $\mathrm{R}$ of obtaining (at least) some information by counting on the performance of $\mathrm{S}$, and the absence of supervision on $\mathrm{S}$ ' performances, which means that $\mathrm{R}$ does not verify the truthfulness of $S$ ' messages, nor does she verify how $S$ elaborated the transmitted information. These aspects are peculiar to the incidence of trust and can be explained if one

\footnotetext{
${ }^{8}$ http://www.silk-road.com/artl/marcopolo.shtml

${ }^{9}$ The indirect relation between the level of trust and the level of resources falls under the mini-max rule. On the basis of the latter, trust and trustor's resources are variables of the same system, and they are correlated in such a way that the growth of one determines the decrease of the other. For a more in-depth analysis of the relation between trust and trustor's resources see (Taddeo, 2010).

${ }^{10}$ See (Taddeo, 2010) for the analysis in support of this definition.
} 
considers trust as a property qualifying the communication of information in a testimony scenario. Following this analysis, testimony can be defined thus (Figure 2):

Testimony: Assume a first-order relation of communication, where some semantic information $i$ is passed from a sender to a receiver. If the communication occurs between a receiver and a trustworthy sender, and if the receiver acquires the sender's messages without checking their truthfulness, then the communication is affected by the second-order property of trust. Such an occurrence of communication is called testimony.

\section{INSERT FIGURE 2 HERE}

Figure 2 The link between testimony and trust. Given a communication occurring between a sender and a receiver of a message, if the communication is affected by trust then testimony occurs.

The analysis presented in this section concludes the second phase of the solution process of the KA problem. We should now focus on the third phase and address the main issue, namely under which circumstances a receiver acquires some knowledge through the testimony of a sender.

\section{Floridi's Network Theory of Account and Testimony}

According to the analysis presented so far, M conveys an instance of information (understood as well-formed, meaningful and veridical data) and trust facilitates the communication process, but it does not affect the epistemic process ongoing in a testimony scenario. We can rephrase the KA problem on the basis of this analysis as the problem concerning the possibility for the receiver to acquire any knowledge, given the information communicated through the sender's testimony.

Once put in this way, the KA problem points to another fundamental issue, that is, whether information provides a sufficient ground for knowledge. (Floridi, Forthcoming) addresses and solves this issue by showing how information can be upgraded to knowledge using the NTA. In doing so, Floridi provides a substantive analysis of knowledge, according to which knowledge is defined as "accounted semantic information" (page to be defined). In the rest of this section, I will describe only the most fundamental aspects of the NTA and I will show how such a theory can be used to solve the KA problem. The reader interested in learning more about the NTA is referred to (Floridi, Forthcoming).

The NTA is grounded on two pillars, the definition of (semantic) information as wellformed, meaningful and veridical data, and an information flow network able to account for the information held by the epistemic agent. 
I have already presented the definition of information in section two. The time has come to consider the epistemic implication of such a definition. According to Floridi "semantic information is, strictly speaking, inherently truth-constituted and not a contingent truth-bearer [...]" (Floridi, Forthcoming) [added emphasis]. The veridical nature of information deeply connects information and knowledge, because the truthfulness of information corresponds to the truthfulness of knowledge. This means that information provides the basis for the achievement of knowledge because it provides the truth of which the epistemic agent will eventually acquire some knowledge.

However, the truthfulness of information is a necessary but not yet sufficient criterion to upgrade information to knowledge. In order to be upgraded, an instance of information needs also to be accounted for. Before considering in more details how such an account should be provided, I shall focus on the relation between information and knowledge.

A standalone piece of information $p$ is like a thread pulled away from the fabric of interrelations to which it belongs. It is an instance of true semantic data, but it is deprived " $[\ldots]$ of its potential role as evidence and of its potential value for prediction or retrodiction, inferential processes and explanation" (Floridi, Forthcoming). $p$ can gain such potential role and values and be upgraded to knowledge only when it is embedded in the right network of conceptual interrelations that can provide reasons in its support. In such a network, different instances of information are coherently held together, account for each other and yield an "integrated picture of the world", (Dummett, 2004).

Let us consider one more time the case of Sam and Robert. Suppose that Robert has been given by Sam the information $p$ that Radium was discovered in 1898 , and that this is all the information that Robert holds about the discovery of Radium. Robert cannot give any reason for holding that $p$. This is why he is informed that $p$ but he does not have any knowledge that $p$. The only way for Robert to achieve such knowledge is to provide reasons in support that $p$, that is to explain how come that $p$ is the case. Only when Robert is able to provide such an explanation can he upgrade the information that $p$ to the knowledge that $p .^{11}$

Floridi considers the information that can give reasons in support that $p$, that is, the information that explains the reasons why $p$ is the case to be the answer to what he calls how come questions (HC-questions). Let me clarify. Any standalone instance of information poses a set of HC-questions concerning the path of events that generated the scenario described by $p$

\footnotetext{
${ }^{11}$ The reader should note that the thesis presented in this paper rests on an informational definition of knowledge and not on a doxastic one. Therefore knowledge is defined as accounted semantic information and not as a true, justified belief. For this reason, it would be improper to consider this thesis with respect to the debate on Internalism/Externalism, as the account of knowledge presented here rests on completely different background from the one on which such a debate is grounded. Floridi's theory overcomes this debate by shifting the foundation of knowledge from the doxastic to the informational ground.
} 
(genealogical HC-questions), the mechanism or the way in which the scenario described in $p$ was determined (functional $\mathrm{HC}$-questions), and the purpose to which the scenario described in $p$ is devoted (teleological HC-questions). The information conveyed by the answers to such questions provides the explanation for $p$. The HC-questions are posed by $p$ and are answered by an information source (S). The information is transmitted from $\mathrm{S}$ to $p$ indirectly, through some 'relays', which mediate between $\mathrm{S}$ and $p$. The relays, $\mathrm{S}, p$ and the links among them form a network in which the information flows from $\mathrm{S}$ to $p$ via the relays. Such a network accounts for $p$.

Floridi refers to the Network Theory developed in applied mathematics, see (Ahuja, Magnanti, \& Orlin, 1993) and (Newman, Barabási, \& Watts, 2006), to model the information flow network, which answers the HC-questions posed by $p$ and accounts for it.

Let me briefly illustrate how the information flow network works. ${ }^{12} p$ poses a number of HC-questions, these questions are transformed in Boolean HC-questions. The questions to be answered are of the kind of 'was Radium discovered in 1898 because scientists were working on radioactive elements at that time?', 'was Radium discovered in 1898 because that was the time scientists first acquired the adequate lab equipment?', and 'was radium discovered in 1898 because around 1898 European scientists wanted to discover new medical techniques?'. ${ }^{13}$ The answers to these questions are provided by $\mathrm{S}$, which is indirectly connected to $p$. The communication between $\mathrm{S}$ and $p$ is mediated by the relays (vertices in the network) as shown in Figure 3. The vertices might be witnesses, documents, news that convey the information provided by S. In the case of our example, S could be Madam Curie who held some information about the discovery of Radium, and such information is transmitted through her diaries, journals, articles, interviews and so on.

\section{INSERT FIGURE 3 HERE}

Figure 3 Floridi's example of flow network. The network is a finite directed graph G, and represents the pattern of relations that link $\mathrm{S}$ and $\mathrm{t}(\mathrm{p}$ in the example provided in this paper). The edges represent the communication channels. $\mathrm{c}$ indicates the set capacity, that is how much information they channels can possibly convey, while f is the actual flow, i.e. the amount of information that they actually convey. The path from $\mathrm{S}$ to $\mathrm{t}$ is mediated by the nodes (V, vertices), which relay the information. Illustration from (Floridi, Forthcoming).

Now that the NTA has been described we can turn our attention to its implementation to solve the KA problem.

In a testimony scenario, $M$ is usually a standalone piece of information. As such, $M$ comes with an informational deficit, which will be satisfied by the answers to the Boolean HC-questions that it poses. In order to achieve any knowledge of M, R needs to connect such questions to their

\footnotetext{
${ }^{12}$ The reader may find more details about the NTA and the information flow network in (Floridi, Forthcoming).

${ }^{13}$ (Goldsmith, 2006)
} 
correct answers. As showed by the NTA, the answers are transmitted through a network that will provide an account for $\mathrm{M}$. Once accounted, $\mathrm{M}$ will be upgraded to knowledge.

Let us consider how the NTA applies to the testimony case described in our example. Sam communicates to Robert that 'Radium was discovered in 1898', and Robert accept this message as true on the basis of his trust in Sam. At this point Robert is informed that Radium was discovered in 1898. He will know that Radium was discovered in 1898 if and only if he will be able to account for this information. In order to do so, Robert needs to consider the reasons that could explain why it is the case that Radium was discovered in 1898. Soon Robert will find himself in the position to consider the HC-questions (for example, genealogical and teleological HC-questions, among others) posed by $p$ and to seek an answer for them. Suppose the answers will be found in the diaries and in the lab journals of Madam Curie. The information conveyed in these documents shows that in 1898 scientists were working on radioactive elements and that it was in those years that the adequate lab equipment for the measurements of radioactive elements was designed and built.

As Figure 4 illustrates, Robert is informed that $p$ by Sam. $p$ poses a set of HC-questions. Robert seeks the answers to the HC-questions and found them in a network that connects the information provided by the source, Madam Curie, who conveys some first-hand information via some relays, her diaries and the lab journals, to Robert. The information flow forms a network that accounts for $p$.

\section{INSERT FIGURE 4 HERE}

Figure 4 The Information flow network that account for $p$ in Robert's and Sam's testimony scenario.

The NTA and its application to a testimony scenario complete the solution of the KA problem by showing how the information transmitted through testimony can be upgraded to knowledge. It is now time to consider in more detail two aspects of the solution presented in this paper, which may lead to a few potential objections. This is the task of the next section.

\section{The role of the sender and the information flow network}

The analysis presented in this paper unveils two processes ongoing in a testimony scenario, a process of communication, from $\mathrm{S}$ to $\mathrm{R}$, and an upgrading process of the communicated instance of information to knowledge. While the sender of the message plays a fundamental role in the 
communication process, i.e., she is trusted to communicate some well-formed, meaningful and veridical data, as a sender she does not play any role in the upgrading process. ${ }^{14}$ This aspect differentiates the analysis presented in this paper from the ones provided in the literature, according to which the sender of the message is involved in the receiver's process of acquiring some knowledge, as the sender's trustworthiness provides the means for the epistemic justification.

The distinction between the communication and the upgrading processes follows from both the analysis of trust, as a second-order property of first-order relations, and the NTA. The analysis of trust shows that, even when trust is grounded on a rationally assessed trustworthiness, it does not exclude the possibility of epistemic luck and hence that trust does not provide any epistemic justification for R to accept S' message. The NTA provides the means for an epistemic agent, R in the case of a testimony scenario, to achieve some knowledge independently from the source of the message. According to this theory, the achievement of knowledge is entirely due to R's intellectual capacities to provide reasons to account for S' message. This means that $\mathrm{R}$ can achieve knowledge of $\mathrm{M}$, provided that he is able to account for it, even in those circumstances in which $\mathrm{S}$ might have communicated a true message only by chance. It is worth stressing that the two processes are equally necessary to the final purpose of achieving some knowledge through testimony. $\mathrm{R}$ needs both a truthful message and a method to account for it in order to reach his goal, while $\mathrm{S}$ has the fundamental role of communicating the truthful message.

The central role that $\mathrm{R}$ plays in the knowledge achievement process might give rise to a criticism concerning the process of connecting $\mathrm{M}$ to the conceptual network of interrelations to which it belongs. One could object that, in order to do this, R needs to have some background knowledge similar, if not equal, to S'. If this holds, then the whole point of testimony, understood as transmission of knowledge on the basis of S' communication, is lost because, in order to acquire any knowledge, $\mathrm{R}$ does not count on $\mathrm{S}$ ' expertise but has to acquire some expertise by himself. This objection provides the opportunity to consider another aspect of the analysis presented so far.

The same instance of information can be explained (accounted for) in several ways (at several levels of abstractions) depending on the kind of HC-questions for which one seeks an answer. A scientist, for instance, can account for M referring to an informational network based on scientific information, like the place of Radium on Mendeleev's table, and the report of some scientific experiments and so on. The receiver of the message, may not be a scientist, and does not necessarily have to account for the message using the same network of a scientist. He could do so

\footnotetext{
${ }^{14}$ Note that this does not exclude the cases in which $S$ is also part of the information network that accounts for the communicated message. In these cases $\mathrm{S}$ plays two distinct roles, as sender of the message and as a node in the
} 
by referring to another kind of source, an historical one for example, which would offer historical reasons in support of $\mathrm{M}$. Generally speaking, $\mathrm{R}$ can account for M's message referring to a different information network. Such a network may as well convey less specific information than the one to which S refers. Nevertheless, both the networks offer an account for the same instance of information.

Once could argue that, if so, then testimony allows one to achieve only a superficial or indirect kind of knowledge, which might be further qualified as an inferior kind of knowledge if compared to the other kinds of knowledge. Fortunately, the goal of this analysis is to show whether and under which circumstances a receiver acquires some knowledge through the testimony of a sender. The kind and the quality of such knowledge are not at stake in this paper. Therefore, I will not pursue the analysis of the quality of knowledge achieved through testimony, as it falls out of the scope of this paper, but rather move on to consider in detail another critical aspect of the analysis presented here.

The second aspect refers to the use of the information flow network described in the NTA to solve the KA problem. The information flow network is not used to verify the truthfulness of the message transmitted by the sender, but to corroborate it by providing reasons in its support. The dichotomy between the alethic value of the message and the account provided by the network might lead to one more objection, the last that I will consider in this paper. One could claim that, given this dichotomy, the information flow network could be used to account for false messages. In a testimony scenario, this can have serious consequences, because the network could provide reasons for accepting as true a message that is actually false. If this is the case, then the NTA does not provide a valid method to achieve knowledge. On the contrary, it provides a method to support whatever message is communicated, independently from its alethic value.

A clarification is required to defuse this objection. The truthfulness of both the standalone instance of information (M) and of the semantic content transmitted though the network is a necessary requirement in order to forge a network that accounts for the message. Information, which is inherently true, has to be transmitted through the network in order to account for the message. Of course, one could set a network in which false semantic content is communicated, but in this case not information but mis- or dis-information is transmitted, and a mis- or a disinformation network does not provide any account for any message. The reader should recall that NTA assumes the truthfulness of message and that of the data transmitted through the network as a precondition for upgrading information to knowledge. 


\section{Conclusion}

This paper contributes to the debate on testimony by proposing an information-based approach for its analysis. The information-based approach allows for a redefinition of testimony as an occurrence of communication of information (understood as well-formed, meaningful and veridical data) qualified by the second-order property of trust.

Trust is defined as a second-order property that ranges over first-order relations facilitating their occurrences. I argued that the presence of trust explains R's decision to accept S' message as true without verifying it but does not provide an epistemic justification in support of the message. Hence, trust is insufficient to provide all the required conditions for the achievement of knowledge. I have then shown that, in order to accomplish this goal, $\mathrm{R}$ has to upgrade the communicated information to knowledge, by providing an account for it, that is, by offering reasons in support of the communicated instance of information. I referred to Floridi's NTA to show how such an account can be modelled.

On the basis of this analysis, the puzzle of the relation between trust and testimony has been solved, by showing that trust has a fundamental role in testimony scenario, as it facilitates the communication between $\mathrm{S}$ and $\mathrm{R}$, but that trust does not provide the means through which $\mathrm{R}$ can achieve some knowledge. The KA problem has been solved by showing how the message conveyed in a testimony scenario can allow the receiver to achieve some knowledge.

\section{Acknowledgements}

A previous draft of this paper has been presented at the workshop on "Philosophy of the Information and Computing Sciences" held at the Lorentz Center, The Netherlands. I am very grateful to the participants to the workshop for their valuable comments, as well as to the referees of this paper and to James Collier for his assistance throughout the editing process. They are responsible only for the improvements not for any remaining mistake.

\section{References}

Ahuja, R. K., Magnanti, T. L., \& Orlin, J. B. (1993). Network Flows : Theory, Algorithms, and Applications Englewood Cliffs, N.J: Prentice Hall.

Antony, L. M., \& Witt, C. (1993). A Mind of One's Own: Feminist Essays on Reason and Objectivity: Boulder: Westview Press.

Audi, R. (1997). The Place of Testimony in the Fabric of Justification and Knowledge. American Philosophical Quarterly, 34, 405-422.

Baier, A. (1994). Sustaining Trust. In A. Baier (Ed.), Moral Prejudices (pp. 152-182): Harvard University Press. 
Baier, A. (1995). Trust and Its Vulnerabilities Moral Prejudices: Essays on Ethics. Cambridge, MA: Harvard University Press.

Bar-Hillel, Y., \& Carnap, R. (1953). An Outline of a Theory of Semantic Information. repr. in BarHillel [1964], 221-274.

Blais, M. J. (1987). Epistemic Tit for Tat. Journal of Philosophy, 84, 363-375.

Burge, T. (1997). Interlocution, Perception, and Memory. Philosophical Studies, 86 21-47.

Coady, C. A. J. (1992). Testimony : a philosophical study. Oxford: Clarendon Press.

Colburn, T. R. (2000). Information, Thought, and Knowledge. Paper presented at the Proceedings of the World Multiconference on Systemics, Cybernetics and Informatics.

Devlin, K. J. (1991). Logic and Information. Cambridge: Cambridge University Press.

Dretske, F. I. (1981). Knowledge and the Flow of Information. Oxford: Blackwell. Reprinted in 1999 (Stanford, CA: CSLI Publications).

Dummett, M. A. E. (2004). Truth and the Past. New York; Chichester: Columbia University Press.

Floridi, L. (2004). Outline of a Theory of Strongly Semantic Information,. Minds and Machines, 14(2), 197-222.

Floridi, L. (2005). Is Information Meaningful Data? Philosophy and Phenomenological Research, $70(2), 351-370$.

Floridi, L. (Forthcoming). Semantic Information and the Network Theory of Account. Synthese.

Fricker, E. (1995). Telling and Trusting: Reductionism and Anti-Reductionism in the Epistemology of Testimony. Mind and Society, 104, 393-411.

Gambetta, D. (1998). Can We Trust Trust? Trust: Making and Breaking Cooperative Relations. In D. Gambetta (Ed.), Trust: Making and Breaking Cooperative Relations (pp. 213-238). Oxford: Basil Blackwell.

Goldberg, S. C. (2001). Testimonially based knowledge from false testimony. The Philosophical Quarterly, 51, 512-526.

Goldman, A. I. (1999). Knowledge in a Social World. Oxford: Oxford University Press.

Goldsmith, B. ( 2006). Obsessive Genius: The Inner World of Marie Curie London: W. W. Norton \& Co.

Govier, T. (1993). Trust and Testimony: Nine Arguments on Testimonial Knowledge. International Journal of Moral and Social Studies, 8, 21-39.

Govier, T. (1997). Social Trust and Human Communities. Montreal: McGill-Queens University Press.

Graham, P. J. (1997). What is testimony? Philosophical Quarterly, 47, 227-232.

Graham, P. J. (2000). Transferring Knowledge. Nous, 34(1), 131-152.

Grice, H. P. (1989). Studies in the Way of Words Cambridge, Mass.: Harvard University Press.

Hardwig, J. (1991). The Role of Trust in Knowledge. The Journal of Philosophy(88), 693-708.

Hinchman, E. (2005). Telling as inviting to trust. Philosophy and Phenomenological Research., 70 (3), 562-587.

Holton, R. (1994). Deciding to trust, coming to believe. Australasian Journal of Philosophy, 72, 6376.

Jones, K. (1999). Second-Hand Moral Knowledge. The Journal of Philosophy, 96, 55-78.

Lackeyand, J., \& Sosa, E. (Eds.). (2006). The Epistemology of Testimony. Oxford: Oxford University Press.

Lagenspetz, O. (1992). Legitimacy and Trust. Philosophical Investigations, 15(1), 1-21.

Littlejohn, S. W. T. (2002). heories of human communication. Wadsworth: 7th edition, Belmont.

McDowell, A. (2002). Trust and information: the role of trust in the social epistemology of information science. Social Epistemology, 16(1), 51-63.

Newman, M. E. J., Barabási, A.-L., \& Watts, D. J. (Eds.). (2006). The Structure and Dynamics of Networks. Princeton, N.J: Princeton University Press.

Pritchard, D. (2004). The Epistemology of Testimony. Philosophical Studies, 14, 326-348.

Schmitt, F. (1987). ustification, Autonomy, Sociality. Synthese, 73, 43-85. 
Taddeo, M. (2009). Defining Trust and E-trust: Old Theories and New Problems,. nternational Journal of Technology and Human Interaction (IJTHI), 5(2), 23-35.

Taddeo, M. (2010). Modelling Trust in Artificial Agents, a First Step toward the Analysis of eTrust. Minds and Machines, 20(2), 243-257.

Welbourne, M. (1981). The Community of Knowledge. The Philosophical Quarterly, 31, 302-314.

Welbourne, M. (1993). The Community of Knowledge: Atlantic Highlands: The Humanities Press. 